\title{
Visible Light Induced Photocatalytic Degradation of Methyl Red with Codoped Titania
}

\author{
Susmitha Thota, ${ }^{1}$ Siva Rao Tirukkovalluri, ${ }^{1}$ and Sreedhar Bojja ${ }^{2}$ \\ ${ }^{1}$ Department of Inorganic \& Analytical Chemistry, School of Chemistry, Andhra University, Visakhapatnam 500 003, India \\ ${ }^{2}$ Department of Chemistry, CSIR-Indian Institute of Chemical Technology, Hyderabad 500 007, India \\ Correspondence should be addressed to Siva Rao Tirukkovalluri; sivaraotvalluri.16@gmail.com
}

Received 15 June 2014; Revised 15 September 2014; Accepted 29 September 2014; Published 20 October 2014

Academic Editor: Adel A. Ismail

Copyright (c) 2014 Susmitha Thota et al. This is an open access article distributed under the Creative Commons Attribution License, which permits unrestricted use, distribution, and reproduction in any medium, provided the original work is properly cited.

\begin{abstract}
Photocatalysis using semiconductor oxides was being investigated extensively for the degradation of dyes in effluent water. This paper reports our findings on visible light induced photocatalytic degradation of azo dye, methyl red mediated nitrogen and manganese codoped nano-titanium dioxide $\left(\mathrm{N} / \mathrm{Mn}-\mathrm{TiO}_{2}\right)$. The codoped samples with varying weight percentages were synthesized by sol-gel method and characterized by various analytical techniques. The X-ray diffraction data showed that the synthesized samples were in anatase phase with $2 \theta$ at $25.3^{\circ}$. UV-visible diffuse reflectance spectral analysis revealed that the presence of dopants in $\mathrm{TiO}_{2}$ caused a significant absorption shift towards visible region and their presence was confirmed by X-ray photoelectron spectral data. The release of hydroxyl radical (major active species in photocatalytic degradation) by the photocatalyst in aqueous solution under visible light irradiation was quantitatively investigated by the photoluminiscent technique (PL). The effect of various experimental parameters like dopant concentration, $\mathrm{pH}$, catalyst dosage, and initial dye concentrations was investigated and optimum conditions were established. The extent of mineralization of methyl red was studied by chemical oxygen demand (COD) assays and the results showed complete mineralization of the dye.
\end{abstract}

\section{Introduction}

Dyes are the resistant compounds that are found in industrial waste water causing adverse environmental problems. Most of the dyes used in the pigmentation of textiles, leather, paper, ceramics, and food-processing are derived from azo dyes. Approximately $15 \%$ of these are lost with waste water during synthesis and processing [1]. This represents a great hazard to human and environmental health due to the toxicity of azo dyes [2]. The treatment of such pollutants can be achieved by heterogeneous photocatalysis due to its efficiency and low cost as well as to the fact that it allows complete degradation of pollutants to carbon dioxide and inorganic acids [3].

$\mathrm{TiO}_{2}$ has attracted a great deal of study for its high photocatalytic activity. But the fatal drawbacks of $\mathrm{TiO}_{2}$ are its wide band gap and high rate of electron-hole $\left(\mathrm{e}^{-} / \mathrm{h}^{+}\right)$ recombination [4]. A number of studies have been reported on the modification of $\mathrm{TiO}_{2}$ in order to extend the absorption of light to the visible region. These include dye sensitization, semiconductor coupling, impurity doping, use of coordination metal complexes, and metal deposition [5]. Among all these methods, codoping $\mathrm{TiO}_{2}$ with a metal and nonmetal is found to be most suitable. According to literature survey, the nonmetal dopant reduces the band gap making it active in visible region and metal dopant facilitates the charge separation of $\mathrm{e}^{-} / \mathrm{h}^{+}$and thus decreases their rate of recombination [6]. Besides, considering the significance of visible light responsive photocatalyst, it is very interesting to search for new photocatalyst with appropriate crystal phase, particle size, and other surface properties to improve the photocatalytic activity. Hence in our research work we have selected nitrogen $(\mathrm{N})$ and manganese $(\mathrm{Mn})$ as nonmetal and metal dopants to be codoped in $\mathrm{TiO}_{2}$ and study its photocatalytic activity by degrading a model azo dye pollutant, methyl red.

Various methods available for the synthesis of $\mathrm{TiO}_{2}$ photocatalyst include precipitation [7], hydrothermal, solvothermal [8], chemical vapour deposition [9], and electrospinning [10]. Among several methods available for synthesis of nanopowders, sol-gel method is advantageous because 
powders of homogenous concentrations and high purity can be synthesized at very low temperatures and high purity can be synthesized at low temperature under stoichiometry control [11]. Hence, sol-gel method has been followed for the synthesis of nitrogen and manganese codoped $\mathrm{TiO}_{2}(\mathrm{~N} / \mathrm{Mn}-$ $\mathrm{TiO}_{2}$ ) photocatalysts.

1.1. Synthesis of Photocatalysts. A series of $\mathrm{TiO}_{2}$ samples were prepared by codoping with nitrogen and manganese in the range of $0.25-1.00 \mathrm{wt} \%$ along with undoped $\mathrm{TiO}_{2}$ by solgel method. For preparation of undoped $\mathrm{TiO}_{2}$ ethanol is taken as solvent and water was added to titanium tetrabutoxide (titanium precursor) for hydrolysis and condensation reactions to take place in presence of nitric acid. In case of codoped catalyst preparation, calculated quantities of manganese nitrate and urea, precursors of $\mathrm{Mn}$ and $\mathrm{N}$, were first dissolved in ethanol along with water and the resultant solution was added drop by drop to the ethanol solution of $\mathrm{Ti}(\mathrm{OBu})_{4}$ under vigorous stirring. After complete addition, the colloidal suspension was allowed to stir for $90 \mathrm{~min}$ and aged for $48 \mathrm{hrs}$. Thus obtained gel was dried in an oven at $70^{\circ} \mathrm{C}$. Later, it was well ground and calcined at $400^{\circ} \mathrm{C}$ for about $5 \mathrm{hrs}$ in muffle furnace at the rate of $2^{\circ} / \mathrm{min}$ and then cooled in a desiccator and ground to form homogenous powder.

1.2. Characterisation of Photocatalysts. XRD spectra were recorded for $2 \theta$ from $20^{\circ}$ to $80^{\circ}$ with model Ultima IV, RIGAKU diffractometer using monochromatized $\mathrm{CuK} \alpha$ radiation $(\lambda=1.541 \AA)$ with a Germanium solid state detector. The size of the nanoparticles was recorded with a TECNAI FE12 TEM (Eindhonen, The Netherlands) instrument operating at $120 \mathrm{kV}$. XPS studies were done using PHI quantum ESCA microprobe system model, using AlK $\alpha$ radiation of $250 \mathrm{~W} \mathrm{X}$-ray tube as a radiation source with energy of $1486.6 \mathrm{eV}, 16 \mathrm{~mA} \times 12.5 \mathrm{kV}$ under working pressure lower than $1 \times 10^{-8} \mathrm{Nm}^{-2}$. The fitting of the XPS curves was analyzed with multipack 6.0A software. UV-visible absorption spectra of the samples were obtained by using Shimadzu 3600, UVvisible NIR spectrophotometer using $\mathrm{BaSO}_{4}$ as reference scatter. Photoluminescent spectral analysis was done using Horiba Jobin Fluoro Max-4 instrument with a PMT voltage of $150 \mathrm{~V}$ and slit set both at $2.5 \mathrm{~nm}$.

1.3. Photocatalytic Activity of Catalyst. Dye solution with catalyst was stirred in dark for 20 min to establish adsorptiondesorption equilibrium on the catalyst surface. The reaction mixture was exposed to visible light source of $400 \mathrm{~W}$ high pressure mercury vapour lamp (Osram, India) by placing it $20 \mathrm{~cm}$ away from light source. At certain regular time intervals, aliquots of samples were collected through $0.45 \mu \mathrm{m}$ Millipore syringe filter. The filtrate was subjected to COD analysis for mineralization studies at regular time intervals by using acidic dichromate method with a Bioblock COD analyzer. Also, the filtrate was analyzed on spectrophotometer at a wavelength of $525 \mathrm{~nm}$ for degradation studies of methyl red and at wavelength of $484 \mathrm{~nm}$ for degradation studies of orange II. To have a comprehensive comparison, the reaction environment is maintained the same for all the activity tests. The percentage degradation of dye was calculated from the following equation:

$$
\% \text { of Degradation }=\frac{A_{o}-A_{t}}{A_{o}} \times 100 \text {, }
$$

where $A_{o}$ is initial absorbance of dye solution before degradation and $A_{t}$ is absorbance of dye solution at time $t$.

\section{Results and Discussions}

2.1. X-Ray Diffraction and Transmission Electron Microscopic Analysis (XRD and TEM). The functionality of $\mathrm{TiO}_{2}$ for different applications depends on its crystal phase. For photocatalytic applications anatase phase is preferred. The XRD patterns of all the samples exhibited typical peaks at $2 \theta=25.3^{\circ}, 37.7^{\circ}, 47.9^{\circ}$, and $54.1^{\circ}$, which can be indexed to the (101), (004), (200), and (211) crystal facets of anatase $\mathrm{TiO}_{2}$ (JCPDS File number: 21-1272). From XRD patterns it is evident that calcinations at $400^{\circ} \mathrm{C}$ would result in anatase crystal phase transition.

TEM image of 0.75 wt.\% $\mathrm{N}$ and 0.25 wt.\% $\mathrm{Mn}-\mathrm{TiO}_{2}$ $(\mathrm{NMN}-4)$ was represented in Figure 1 and average particle size was found to be $9 \mathrm{~nm}$. It is in complete agreement with the results of crystallite size calculated by using the Scherer equation and the full width at half maximum of the (101) peak in the XRD patterns of the material. The particle size of the catalyst plays very important role in its photocatalytic activity. Decrease in particle size results in large surface area which is favorable for high photocatalytic activity.

Based on XRD data and trail photocatalytic activity studies, further characterization has been made for NMN-4 which showed best photocatalytic activity.

2.2. Ultraviolet-Visible Diffuse Reflectance Spectroscopic Studies. The UV-DRS spectral data was shown in Figure 2. It was observed that the synthesized codoped samples had a profound effect on its optical response in the visible wavelength range. Band gap for all the synthesized samples were calculated by using the formula $E_{\mathrm{g}}=(1240 / \lambda)$, where $E_{\mathrm{g}}$ is band gap and $\lambda$ is wavelength [12]. The band gap of synthesized undoped $\mathrm{TiO}_{2}$ was found to be $3.18 \mathrm{eV}$ which is comparable with the literature value [13]. The codoped samples showed band gap ranging from 2.87 to $3.10 \mathrm{eV}$. The band gap of NMN-4 was found to be $2.87 \mathrm{eV}$ which has been reduced by $0.31 \mathrm{eV}$ when compared with that of undoped sample. The narrowing of band gap is maybe due to formation of midgap band above valence band by $\mathrm{N} 2 \mathrm{p}$ states. The electrons are transferred from midgap band to conduction band. Thus by narrowing the band gap, the absorbance has shifted from ultraviolet to visible region. Red shift indicates more photogenerated $\mathrm{e}^{-} / \mathrm{h}^{+}$pairs that could be excited by photons with less energy, leading to better photocatalytic degradation efficiency in visible region. Thus, the results demonstrated that all the synthesized codoped samples had reduced band gap and are active in visible region. 


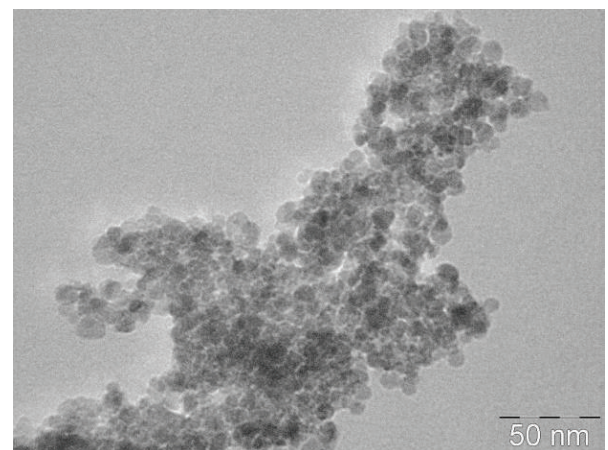

FIGURE 1: TEM image of NMN-4 illustrates the formation of nanoparticles with average particle size of $9 \mathrm{~nm}$.

2.3. X-Ray Photoelectron Spectroscopic (XPS) Analysis. XPS measurements of NMN-4 were carried out to confirm the presence of $\mathrm{N}$ and $\mathrm{Mn}$ and investigate their chemical state. High resolution XPS spectra of NMN-4 with elemental analysis of Ti, O, N, and $\mathrm{Mn}$ were represented in Figure 3. In codoped sample, doublet peaks of $\mathrm{Mn} 2 \mathrm{p}_{3 / 2}$ and $\mathrm{Mn} 2 \mathrm{p}_{1 / 2}$ were observed with binding energies of 641.9 and $653.9 \mathrm{eV}$, respectively. The major peak located at $641.9 \mathrm{eV}$ is attributed to $\mathrm{Mn}^{3+}$ with peak area of $75 \%$ and the minor peak with higher binding energy of $653.9 \mathrm{eV}$ can be assigned to a higher oxidation state $\mathrm{Mn}^{4+}$ [14]. Based on the above XPS data, a reasonable assignment is that $\mathrm{Mn}$ exists in both $\mathrm{Mn}^{4+}$ and $\mathrm{Mn}^{3+}$ states substituting some of the $\mathrm{Ti}^{4+}$ in anatase $\mathrm{TiO}_{2}$ lattice giving rise to new electronic interactions. As $\mathrm{Mn}^{4+} / \mathrm{Mn}^{3+}$ can accept electrons and act as electron trap centre, its doping reduces the rate of $\mathrm{e}^{-} / \mathrm{h}^{+}$recombination. Coming to the $\mathrm{N}$, the binding energy peaks occurred at $399.6 \mathrm{eV}$. This can be typically assigned as interstitial nitrogen dopant [15]. The XPS spectra of undoped $\mathrm{TiO}_{2}$ show spinorbit doublet peaks of $\mathrm{Ti} 2 \mathrm{p}_{3 / 2}$ and $2 \mathrm{p}_{1 / 2}$ located at 459.0 and $464.7 \mathrm{eV}$ which has peak separation of $5.7 \mathrm{eV}$ indicating the presence of $\mathrm{Ti}^{4+}$ in the synthesized nanopowders [16]. The binding energy of Ti $2 \mathrm{p}_{3 / 2}$ of codoped sample shifted to a more positive value by $0.7 \mathrm{eV}$ when compared to that of undoped $\mathrm{TiO}_{2}$ that has binding energy of $458.3 \mathrm{eV}$. Thus the presence of dopants has certain influence on $\mathrm{Ti}^{4+}$ pulling the electrons in $\mathrm{Ti}-\mathrm{O}-\mathrm{Ti}$ bond, a bit away from $\mathrm{Ti}$ atom, thus causing a little rise in $\mathrm{Ti} 2 \mathrm{p}_{3 / 2}$ binding energy. Thus XPS data has confirmed that there is a substitutional doping of $\mathrm{Mn}$ and interstitial doping of $\mathrm{N}$ in $\mathrm{TiO}_{2}$ network.

2.4. Photoluminescent Spectral Studies. Hydroxyl radical is deemed to be one of the crucial reactive species during photocatalytic reaction and is responsible for oxidative decomposition of pollutants. Photoluminescence technique is one of the methods available to investigate the production of ${ }^{\bullet} \mathrm{OH}$. Coumarin is used as a fluorescent probe, which on reaction with ${ }^{\circ} \mathrm{OH}$ results in the formation of 7-hydroxy coumarin [17]. In this technique, $1.0 \mathrm{~g} / \mathrm{L}$ of catalyst is dispersed in $10 \mathrm{mg} / \mathrm{L}$ of coumarin solution in acidic conditions and exposed to visible light radiation. For every $30 \mathrm{~min}$ the reaction solution was filtered and photoluminescent intensity

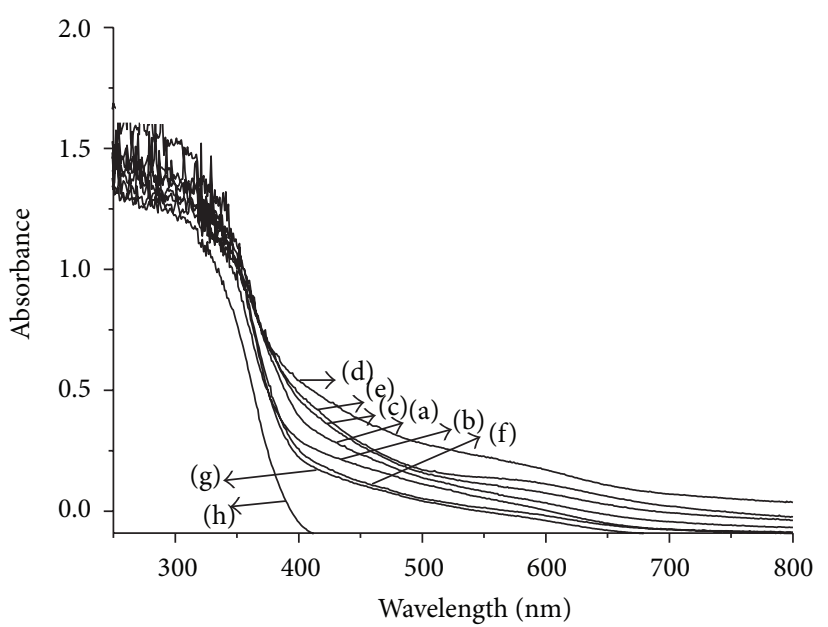

(a) 0.25 wt. $\% \mathrm{~N}$ and $1.00 \mathrm{wt} . \% \mathrm{Mn}-\mathrm{TiO}_{2}$

(b) 0.25 wt. $\% \mathrm{~N}$ and $0.75 \mathrm{wt} . \% \mathrm{Mn}-\mathrm{TiO}_{2}$

(c) $0.50 \mathrm{wt} . \% \mathrm{~N}$ and $0.50 \mathrm{wt} . \% \mathrm{Mn}-\mathrm{TiO}_{2}$

(d) 0.75 wt. $\% \mathrm{~N}$ and 0.25 wt. $\% \mathrm{Mn}-\mathrm{TiO}_{2}$

(e) $1.00 \mathrm{wt} . \% \mathrm{~N}$ and $0.25 \mathrm{wt} . \% \mathrm{Mn}-\mathrm{TiO}_{2}$

(f) $1.00 \mathrm{wt} . \% \mathrm{~N}$ and $0.50 \mathrm{wt} . \% \mathrm{Mn}-\mathrm{TiO}_{2}$

(g) 0.50 wt.\% $\mathrm{N}$ and $1.00 \mathrm{wt} . \% \mathrm{Mn}-\mathrm{TiO}_{2}$

(h) Undoped $\mathrm{TiO}_{2}$

FIGURE 2: UV-visible spectrum of all the synthesized nanocatalysts exhibiting red shift.

was measured from 350 to $600 \mathrm{~nm}$. Figure 4 represents the photoluminescent spectra of the generated 7-hydroxy coumarin with maximum absorption at $450 \mathrm{~nm}$. A linear increase in photoluminescent intensity was observed with increasing irradiation time. However, no maximum absorption is observed for the sample in absence of irradiation $(0 \mathrm{~min})$. It could be inferred that the produced ${ }^{\circ} \mathrm{OH}$ at the catalyst surface was proportional to the irradiation time. The results further confirmed that the synthesized sample NMN4 showed enhanced rate of formation of ${ }^{\circ} \mathrm{OH}$.

2.5. Photocatalytic Degradation of Methyl Red and Optimization of Parameters. Photocatalytic degradation of methyl red was performed by following the procedure given in Section 1.3. A blank test with no photocatalyst is also employed. There is no significant change in the concentration of dye. The high photocatalytic performance is dependent on the comprehensively competing effects of the dopant concentration, $\mathrm{pH}$, initial concentration of pollutant, and catalyst dosage. For practical application in industries, degradation of dyes must be cost effective. Hence, these parameters have to be optimized.

2.5.1. Effect of Dopant Concentration. Photocatalytic degradation studies of different synthesized catalysts with various dopant concentrations on methyl red are presented in Figure 5(a). All the codoped samples showed higher photocatalytic activity than that of Commercial Titania, Degussa P25, under visible light irradiation. So, it is evident that codoping has improved the photocatalytic performance of 

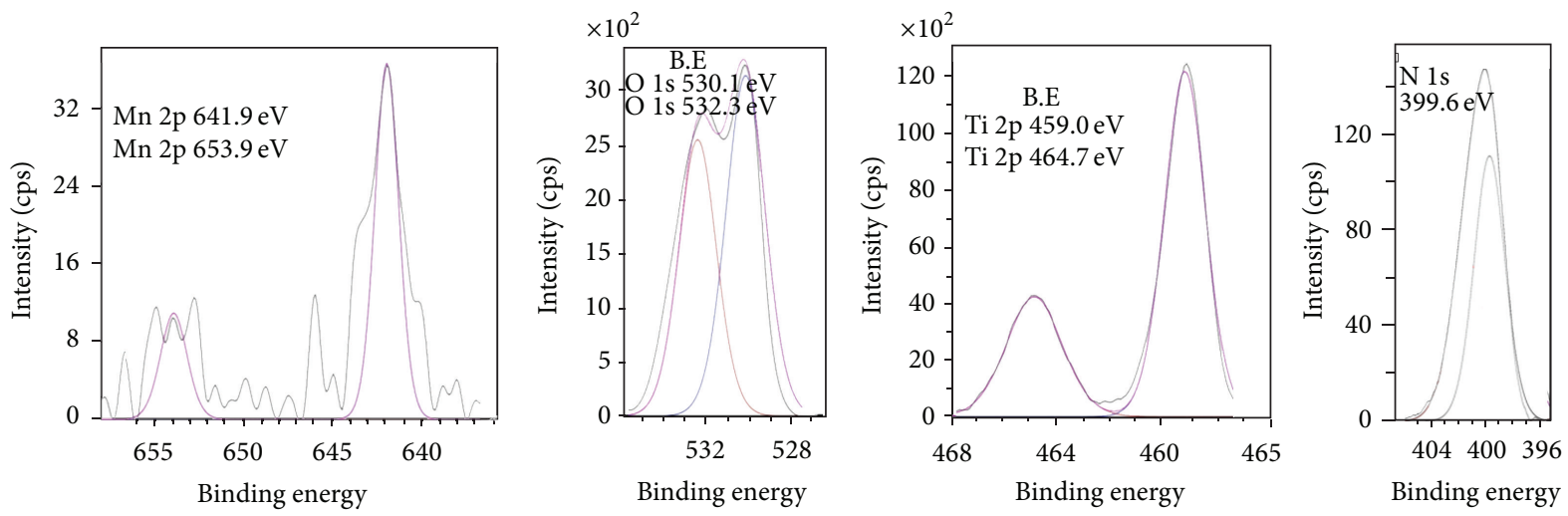

Figure 3: High resolution XPS of the 0.75 wt. $\% \mathrm{~N}$ and $0.25 \mathrm{wt} . \% \mathrm{Mn}-\mathrm{TiO}_{2}(\mathrm{NMN}-4)$.

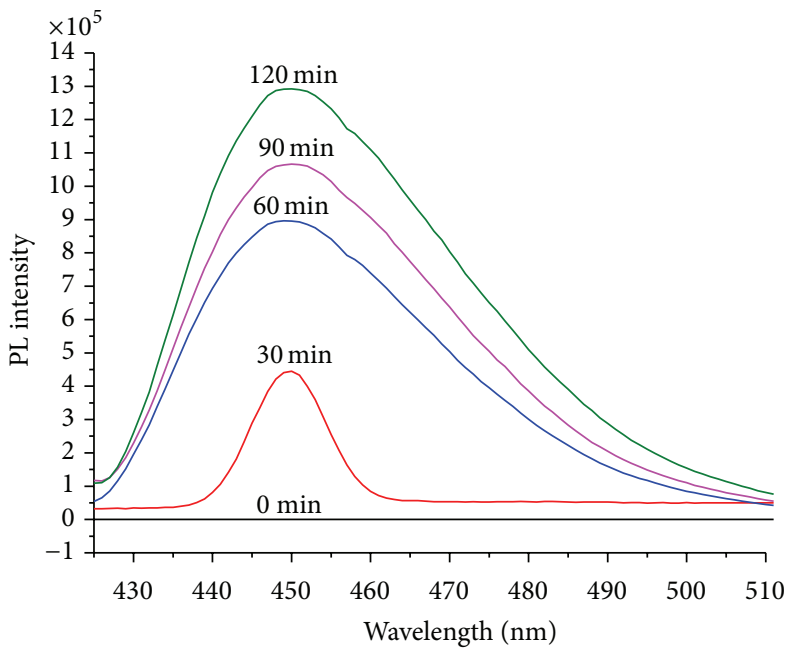

FIGURE 4: PL spectra of NMN-4 indicating the release of $\mathrm{OH}$ radicals when exposed to visible light. The rate of release is very high in first $60 \mathrm{~min}$ and then comparitively decreased.

$\mathrm{TiO}_{2}$. Among all the nanocatalysts, NMN-4 showed highest percentage of degradation. The apparent rates were estimated from the slopes of individual curves. Based on the above analysis, a reasonable explanation of the optimal $\mathrm{N}$ and $\mathrm{Mn}$ content is that, with over dosage of doped content in $\mathrm{TiO}_{2}$, the number of $\mathrm{e}^{-} / \mathrm{h}^{+}$recombination centers will increase which results in low photocatalytic activity.

2.5.2. Effect of $p H$. Figure 5(b) shows the percentage degradation of methyl red as function of time at different $\mathrm{pH}$ values. The sample exhibiting best photocatalytic performance $(\mathrm{NMN}-4)$ was selected and further experiments were conducted to find out the optimum $\mathrm{pH}$ at constant dye concentration $(10 \mathrm{mg} / \mathrm{L})$ and catalyst weight $(1.0 \mathrm{~g} / \mathrm{L})$. It can be seen from the figure that photodegradation of this anionic dye was faster at $\mathrm{pH} 4$. During a photocatalytic oxidation, electrostatic interactions between a semiconductor surface, substrate, and charged radicals strongly depend on the $\mathrm{pH}$ of the solution. In acidic environment, positive charge on $\mathrm{TiO}_{2}$ surface increases which enhances the adsorption of dye molecules on the surface of catalyst. In addition, acidic medium minimizes the $\mathrm{e}^{-} / \mathrm{h}^{+}$recombination. The increase of $\mathrm{pH}$ renders $\mathrm{TiO}_{2}$ surface negatively charged and the repulsion could occur with dye molecules leading to low adsorption of molecules and thus decrease the degradation efficiency. So, optimization of $\mathrm{pH}$ maximizes the photocatalytic performance

2.5.3. Effect of Catalyst Dosage. An optimum catalyst concentration must be determined in order to avoid the catalyst wastage and ensure the total absorption of photons. Photocatalytic degradation experiments carried out with varying amounts $(0.25-3.00 \mathrm{~g} / \mathrm{L})$ of NMN-4 at $\mathrm{pH} 4$ are represented in Figure 5(c). The rate of degradation increased linearly with increase in amount of catalyst up to $2.00 \mathrm{~g} / \mathrm{L}$. The increase in catalyst dosage generally increases the number of photons absorbed by the catalyst, which in turn increases the generation of electrons and holes and thus increases the number of hydroxyl radicals. And also, the increase in catalyst dosage increases the number of organic pollutants adsorbed and facilitates the photocatalytic activity. However, after $2.00 \mathrm{~g} / \mathrm{L}$ of catalyst dosage, the photocatalytic activity decreased due to the turbidity, restriction of light penetration, and agglomeration of catalyst particles. The high catalyst dosage significantly affects the dispersion.

2.5.4. Effect of Initial Concentration of Dye. To determine the optimum initial dye concentration at a fixed catalyst dosage and $\mathrm{pH}$, experiments were carried out with different dye concentrations of 1,5 , and $10 \mathrm{mg} / \mathrm{L}$ and results are represented in Figure 5(d). The optimum initial concentration of dye was found to be $5 \mathrm{mg} / \mathrm{L}$ and further increase in dye concentration decreased the rate of degradation. A reasonable explanation is as follows: as the pollutant concentration increases, the corresponding ratio of reactive species $\left({ }^{\circ} \mathrm{OH}\right)$ may not be formed at the fixed catalyst dosage. Limitation of surface active sites for the catalytic reaction may also control the degradation of methyl red.

2.6. Photocatalytic Mineralization of Methyl Red. COD was performed using acidic dichromate method to study the 


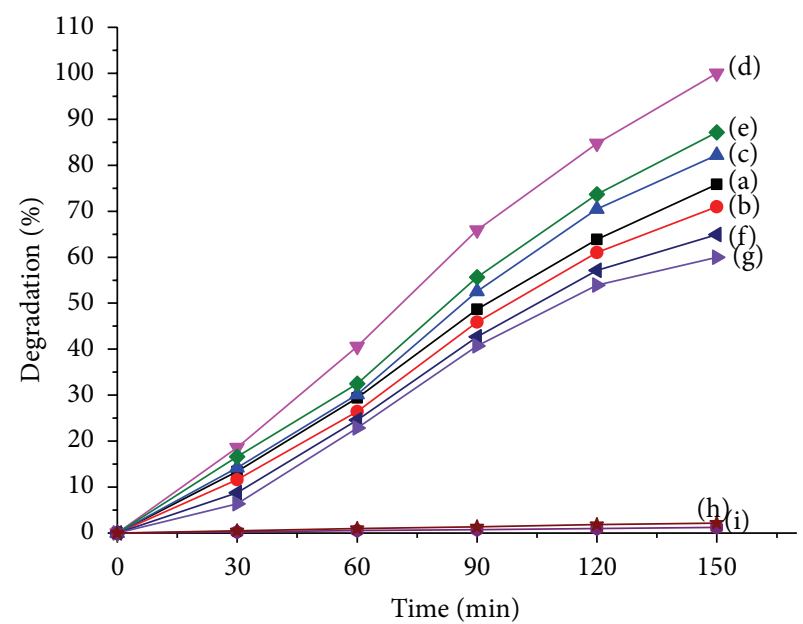
(a) 0.25 wt. $\% \mathrm{~N}$ and 1.00 wt. $\% \mathrm{Mn}-\mathrm{TiO}_{2}$
(b) 0.25 wt. $\% \mathrm{~N}$ and 0.75 wt. $\% \mathrm{Mn}-\mathrm{TiO}_{2}$
(c) 0.50 wt. $\% \mathrm{~N}$ and $0.50 \mathrm{wt} . \% \mathrm{Mn}-\mathrm{TiO}_{2}$
(d) 0.75 wt. $\% \mathrm{~N}$ and 0.25 wt. $\% \mathrm{Mn}-\mathrm{TiO}_{2}$
(e) 1.00 wt. $\% \mathrm{~N}$ and 0.25 wt. $\% \mathrm{Mn}-\mathrm{TiO}_{2}$
(f) $1.00 \mathrm{wt} . \% \mathrm{~N}$ and $0.50 \mathrm{wt} . \% \mathrm{Mn}-\mathrm{TiO}_{2}$
(g) 0.50 wt. $\% \mathrm{~N}$ and 1.00 wt. $\% \mathrm{Mn}-\mathrm{TiO}_{2}$
(h) Undoped $\mathrm{TiO}_{2}$
(i) Degussa P25

(a)

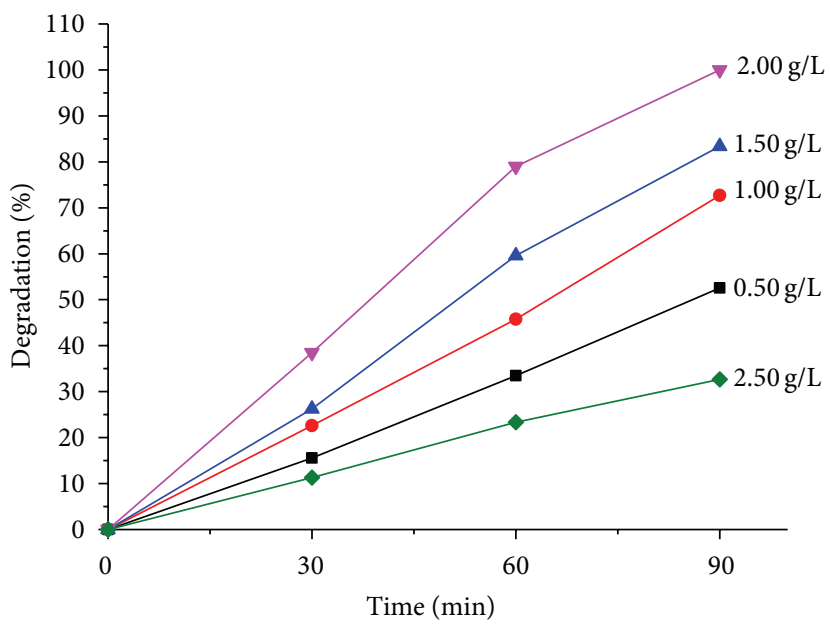

(c)

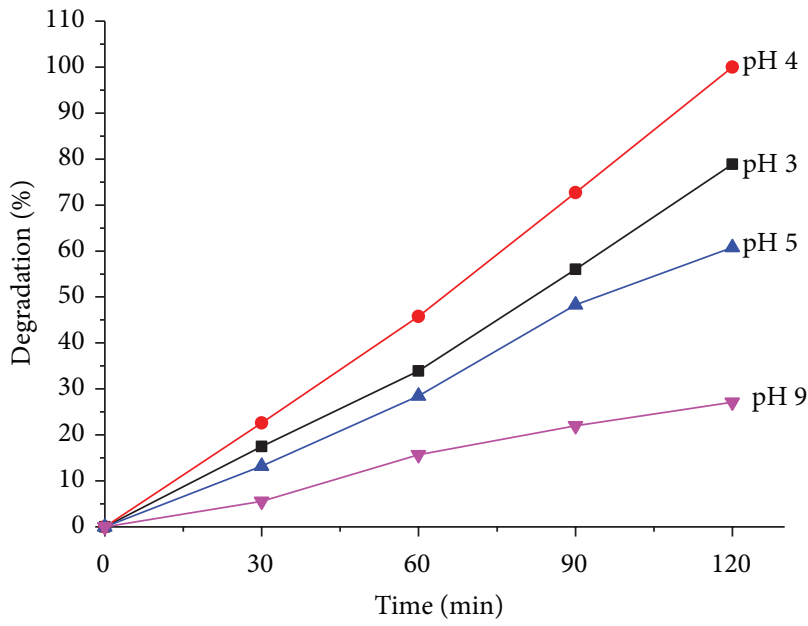

(b)

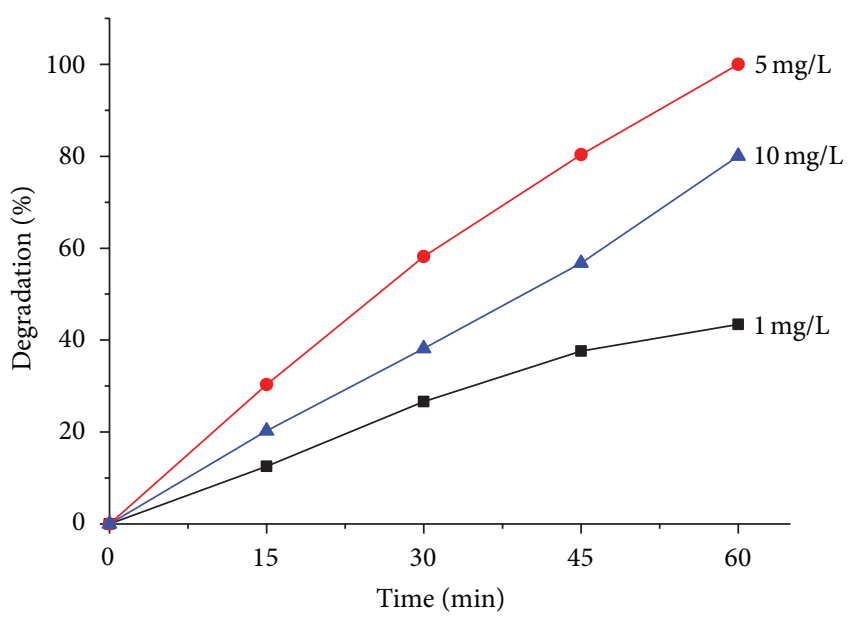

(d)

FIGURE 5: The percentage of degradation of methyl red as function of time by $\mathrm{N} / \mathrm{Mn}-\mathrm{TiO}_{2}$ under different experimental parameters. (a) Effect of Dopant concentration: among all the catalysts with different dopant concentrations, 0.75 wt.\% N and 0.25 wt.\% Mn-TiO 2 (NMN-4) exhibited 100\% degradation in 150 min irradiation time. (b) Effect of $\mathrm{pH}$ : optimum $\mathrm{pH}$ was found to be 4. (c) Effect of catalyst dosage-optimum catalyst dosage was found to be $2.00 \mathrm{~g} / \mathrm{L}$. (d) Effect of initial concentration of dye: $5 \mathrm{mg} / \mathrm{L}$ of methyl red was degraded completely in 60 min by NMN-4 at $\mathrm{pH} 4$ with catalyst dosage of $0.20 \mathrm{~g}$.

mineralization of methyl red. The mineralization experiments were done by treating methyl red $(5 \mathrm{mg} / \mathrm{L})$ with 0.75 wt. $\% \mathrm{~N}$ and 0.25 wt. $\% \mathrm{Mn}-\mathrm{TiO}_{2}(2.00 \mathrm{~g} / \mathrm{L})$ at $\mathrm{pH} 4$ under visible light irradiation. The kinetics of total mineralization of methyl red has been followed by the disappearance of COD. The efficiency of degradation was assessed from the experimentally derived COD, measured at regular intervals of visible light exposure. Figure 6 represents the disappearance of COD with respect to time. It can be seen that there is a gradual reduction in the COD values. However, more time was taken for complete mineralization $(90 \mathrm{~min}$ ) of methyl red when compared with that of degradation $(60 \mathrm{~min})$ under same experimental conditions.

2.7. Photocatalytic Degradation of Orange II. In order to once recheck the photocatalytic activity of the synthesized 


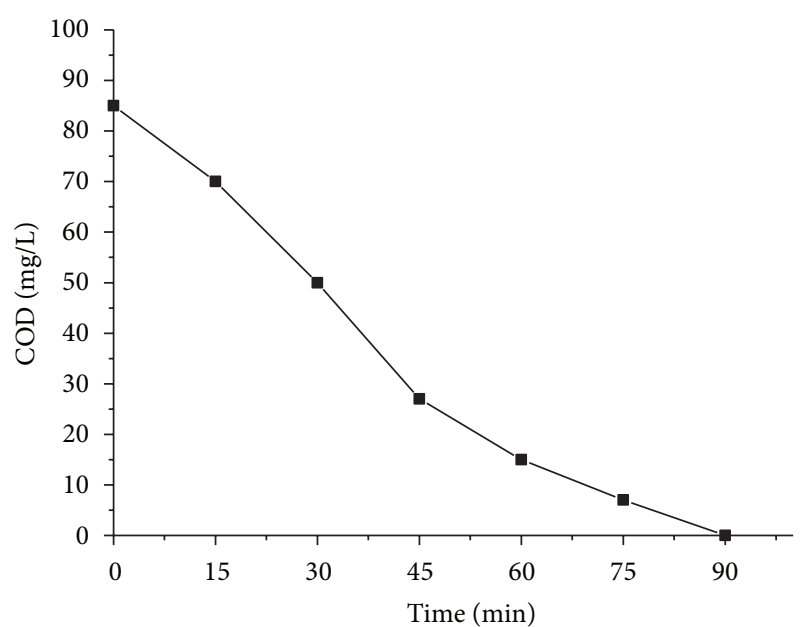

FIGURE 6: Rate of mineralization (COD disappearance) as function of time during degradation of methyl red under visible light. Conditions: $\left[\right.$ methyl red] $=5 \mathrm{mg} / \mathrm{L}, \mathrm{N} / \mathrm{Mn}-\mathrm{TiO}_{2}=2.00 \mathrm{~g} / \mathrm{L}, \mathrm{pH}=$ 4.

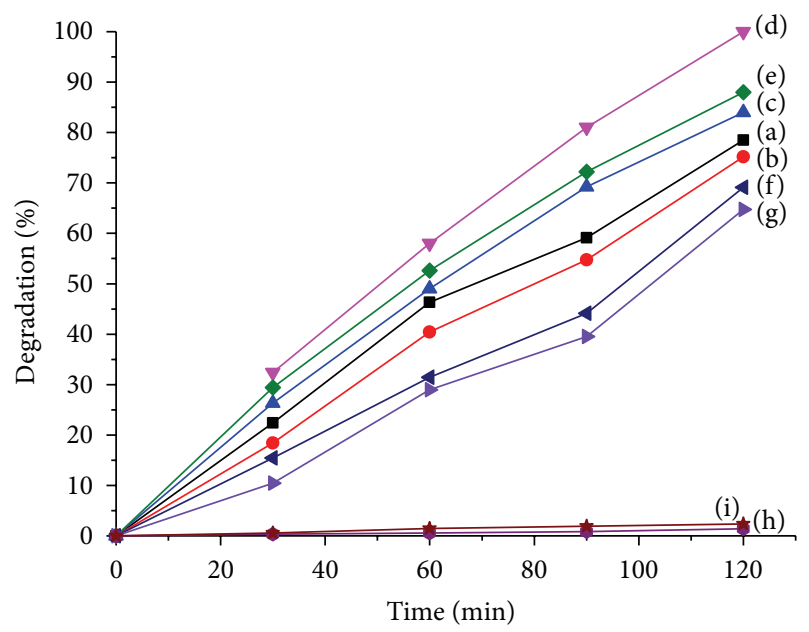
(a) 0.25 wt. $\% \mathrm{~N}$ and 1.00 wt. $\% \mathrm{Mn}-\mathrm{TiO}_{2}$
(b) 0.25 wt. $\% \mathrm{~N}$ and 0.75 wt. $\% \mathrm{Mn}-\mathrm{TiO}_{2}$
(c) 0.50 wt. $\% \mathrm{~N}$ and 0.50 wt. $\% \mathrm{Mn}-\mathrm{TiO}_{2}$
(d) 0.75 wt. $\% \mathrm{~N}$ and 0.25 wt. $\% \mathrm{Mn}-\mathrm{TiO}_{2}$
(e) 1.00 wt. $\% \mathrm{~N}$ and 0.25 wt. $\% \mathrm{Mn}-\mathrm{TiO}_{2}$
(f) 1.00 wt. $\% \mathrm{~N}$ and 0.50 wt. $\% \mathrm{Mn}-\mathrm{TiO}_{2}$
(g) 0.50 wt. $\% \mathrm{~N}$ and 1.00 wt. $\% \mathrm{Mn}-\mathrm{TiO}_{2}$
(h) Degussa P25
(i) Undoped $\mathrm{TiO}_{2}$

Figure 7: Percentage degradation of orange II by photocatalysts with respect to time.

catalysts, a set of experiments were done using another dye, orange II, as target pollutant. The percentage of degradation of dye with respect to time with all the catalysts was represented in Figure 7. In this study also NMN-4 exhibited best photocatalytic activity when compared with the remaining synthesized catalysts. Figure 8 represents the comparative rate of degradation of methyl red and orange II with all the photocatalysts. The rate of degradation is high for orange II

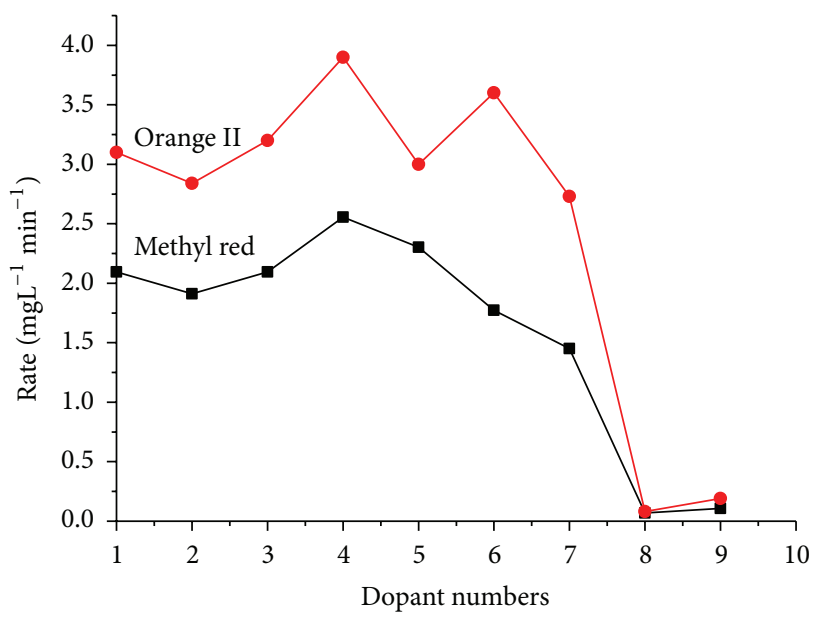

Dopant numbers

(1) 0.25 wt. $\% \mathrm{~N}$ and 1.00 wt. $\% \mathrm{Mn}-\mathrm{TiO}_{2}$

(2) 0.25 wt. $\% \mathrm{~N}$ and 0.75 wt. $\% \mathrm{Mn}-\mathrm{TiO}_{2}$

(3) 0.50 wt. $\% \mathrm{~N}$ and 0.50 wt. $\% \mathrm{Mn}-\mathrm{TiO}_{2}$

(4) 0.75 wt. $\% \mathrm{~N}$ and 0.25 wt. $\% \mathrm{Mn}-\mathrm{TiO}_{2}$

(5) 1.00 wt. $\% \mathrm{~N}$ and 0.25 wt. $\% \mathrm{Mn}-\mathrm{TiO}_{2}$

(6) 1.00 wt. $\% \mathrm{~N}$ and 0.50 wt. $\% \mathrm{Mn}-\mathrm{TiO}_{2}$

(7) 0.50 wt. $\% \mathrm{~N}$ and 1.00 wt. $\% \mathrm{Mn}-\mathrm{TiO}_{2}$

(8) Degussa P25

(9) Undoped $\mathrm{TiO}_{2}$

FIGURE 8: Comparison of degradation rate of methyl red and orange II by photocatalysts.

when compared with that of methyl red. This is maybe due to the presence of sulphate group in dye orange II which assists in photocatalytic degradation. Thus this experiment also proved that $\mathrm{N} / \mathrm{Mn}-\mathrm{TiO}_{2}$ can degrade organic dye pollutants.

\subsection{Mechanism in Release of ${ }^{\circ} \mathrm{OH}$. Consider}

$$
\begin{gathered}
\text { Visible light }+ \text { Catalyst } \longrightarrow \text { Catalyst }\left(\mathrm{h}^{+}+\mathrm{e}^{-}\right) \\
\qquad \begin{array}{c}
\mathrm{h}^{+}+\mathrm{H}_{2} \mathrm{O} \longrightarrow \mathrm{H}^{+}+{ }^{-} \mathrm{OH} \\
\mathrm{h}^{+}+{ }^{-} \mathrm{OH} \longrightarrow{ }^{\circ} \mathrm{OH} \\
\mathrm{e}^{-}+\mathrm{O}_{2} \longrightarrow{ }^{\cdot} \mathrm{O}_{2}{ }^{-} \\
\mathrm{e}^{-}+{ }^{\cdot} \mathrm{O}_{2}{ }^{-}+\mathrm{H}_{2} \mathrm{O} \longrightarrow \mathrm{HO}_{2}{ }^{-}{ }^{-} \mathrm{OH} \\
\mathrm{e}^{-}+\mathrm{HO}_{2}{ }^{-}+\mathrm{H}^{+} \longrightarrow \mathrm{H}_{2} \mathrm{O}_{2} \\
\mathrm{e}^{-}+\mathrm{H}_{2} \mathrm{O}_{2} \longrightarrow{ }^{\cdot} \mathrm{OH}+{ }^{-} \mathrm{OH}
\end{array}
\end{gathered}
$$

\section{Conclusion}

Nitrogen and manganese were successfully doped into $\mathrm{TiO}_{2}$ matrix and its photocatalytic activity was studied for the degradation of methyl red under visible light. All the synthesized samples were in anatase phase and band-gap has been reduced. Nitrogen doping caused visible light response and improved the quantum yield. Manganese doping improved 
the trapping of electrons inhibiting $\mathrm{e}^{-} / \mathrm{h}^{+}$recombination during photocatalytic process. Among all synthesized catalysts 0.75 wt. $\% \mathrm{~N}$ and 0.25 wt. $\% \mathrm{Mn}-\mathrm{TiO}_{2}$ exhibited excellent photocatalytic activity under visible light due to small particle size, large shift in band gap, high crystalline anatase phase, and effective separation of electrons and holes. 100\% degradation of methyl red with initial concentration of $5 \mathrm{mg} / \mathrm{L}$ was achieved in $90 \mathrm{~min}$ at $\mathrm{pH} 4$ with catalyst dosage of $2.00 \mathrm{~g} / \mathrm{L}$. The experimental results revealed that methyl red can be effectively degraded by $\mathrm{N} / \mathrm{Mn}-\mathrm{TiO}_{2}$ without generation of any hazardous wastes or by-products as evident from the considerable reduction in COD. All the photocatalysts also showed excellent catalytic activity on another dye orange II exhibiting complete degradation. It can be concluded that codoping $\mathrm{TiO}_{2}$ with nitrogen and manganese is a promising route for enhanced photocatalytic degradation of pollutants.

\section{Conflict of Interests}

The authors declare that there is no conflict of interests regarding the publication of this paper.

\section{Acknowledgments}

One of the authors, Siva Rao Tirukkovalluri, is highly thankful to University Grants Commission, New Delhi, India, for financial support through Major Research project ((no. F. 39-753/2010 (SR)). One of the authors, Susmitha Thota, is thankful to University Grants Commission, New Delhi, India, for Project Fellow Fellowship.

\section{References}

[1] M. A. Mahmoud, A. Poncheri, Y. Badr, and M. G. Abd El Waned, "Photocatalytic degradation of methyl red dye," South African Journal of Science, vol. 105, no. 7-8, pp. 299-303, 2009.

[2] T. Sauer, G. C. Neto, H. J. José, and R. F. P. M. Moreira, "Kinetics of photocatalytic degradation of reactive dyes in a $\mathrm{TiO}_{2}$ slurry reactor," Journal of Photochemistry and Photobiology A: Chemistry, vol. 149, no. 1-3, pp. 147-154, 2002.

[3] S. K. Kavitha and P. N. Palanisamy, "Photocatalytic and sonophotocatalytic degradation of reactive red 120 using dye sensitized $\mathrm{TiO}_{2}$ under visible light," International Journal of Civil and Environmental Engineering, vol. 3, pp. 1-6, 2011.

[4] C. Kormann, D. W. Bahnemann, and M. R. Hoffmann, "Preparation and characterisation of quantum size titanium dioxide," The Journal of Physical Chemistry, vol. 92, no. 18, pp. 5196-5201, 1988.

[5] N. Hariprasad, S. G. Anju, E. P. Yesodharan, and Y. Suguna, "Sunlight induced removal of Rhodamine B from water through semiconductor photocatalysis: effects of adsorption, reaction conditions and additives," Research Journal of Material Science, vol. 1, pp. 9-17, 2013.

[6] Y. N. Tan, C. L. Wong, and A. R. Mohamed, "An overview on the photocatalytic activity of nano-doped- $\mathrm{TiO}_{2}$ in the degradation of organic pollutants," ISRN Materials Science, vol. 2011, Article ID 261219, 18 pages, 2011.

[7] D. Dvoranova, V. Brezova, M. Mazur, and M. A. Malathi, "Investigations of metal-doped titanium dioxide photocatalysts," Applied Catalysis B: Environmental, vol. 37, pp. 91-105, 2002.
[8] J. Zhu, Z. Deng, F. Chen et al., "Hydrothermal doping method for preparation of $\mathrm{Cr}^{3+}-\mathrm{TiO}_{2}$ photocatalysts with concentration gradient distribution of $\mathrm{Cr}^{3+}$, Applied Catalysis B: Environmental, vol. 62, no. 3-4, pp. 329-335, 2006.

[9] G. Wu, T. Nishikawa, B. Ohtani, and A. Chen, "Synthesis and characterization of carbon-doped $\mathrm{TiO}_{2}$ nanostructures with enhanced visible light response," Chemistry of Materials, vol. 19, no. 18, pp. 4530-4537, 2007.

[10] K. R. Patil, S. D. Sathaye, Y. B. Khollam, S. B. Deshpande, N. R. Pawaskar, and A. B. Mandale, "Preparation of $\mathrm{TiO}_{2}$ thin films by modified spin-coating method using an aqueous precursor," Materials Letters, vol. 57, no. 12, pp. 1775-1780, 2003.

[11] U. G. Akpan and B. H. Hameed, "The advancements in solgel method of doped- $\mathrm{TiO}_{2}$ photocatalysts," Applied Catalysis A: General, vol. 375, no. 1, pp. 1-11, 2010.

[12] H. Natori, K. Kobayashi, and M. Takahashi, "Preparation and photocatalytic property of phosphorus-doped $\mathrm{TiO}_{2}$ particles," Journal of Oleo Science, vol. 58, no. 7, pp. 389-394, 2009.

[13] K. R. Reyes-Gil, E. A. Reyes-García, and D. Raftery, "Photoelectrochemical analysis of anion-doped $\mathrm{TiO}_{2}$ colloidal and powder thin-film electrodes," Journal of the Electrochemical Society, vol. 153, no. 7, pp. A1296-A1301, 2006.

[14] Q. R. Deng, X. H. Xia, M. L. Guo, Y. Gao, and G. Shao, "Mn-doped $\mathrm{TiO}_{2}$ nanopowders with remarkable visible light photocatalytic activity," Materials Letters, vol. 65, no. 13, pp. 2051-2054, 2011.

[15] J. Wang, D. N. Tafen, J. P. Lewis et al., "Origin of photocatalytic activity of Nitrogen-doped $\mathrm{TiO}_{2}$ nanobelts," Journal of the American Chemical Society, vol. 131, no. 34, pp. 12290-12297, 2009.

[16] J. F. Moulder, W. F. Stickle, P. E. Sobol, and K. D. Bomben, Handbook of X-Ray Electron Spectroscopy, PerkinElmer, Eden Prairie, Minn, USA, 1992.

[17] H. Czili and A. Horváth, "Applicability of coumarin for detecting and measuring hydroxyl radicals generated by photoexcitation of $\mathrm{TiO}_{2}$ nanoparticles," Applied Catalysis B: Environmental, vol. 81, no. 3-4, pp. 295-302, 2008. 

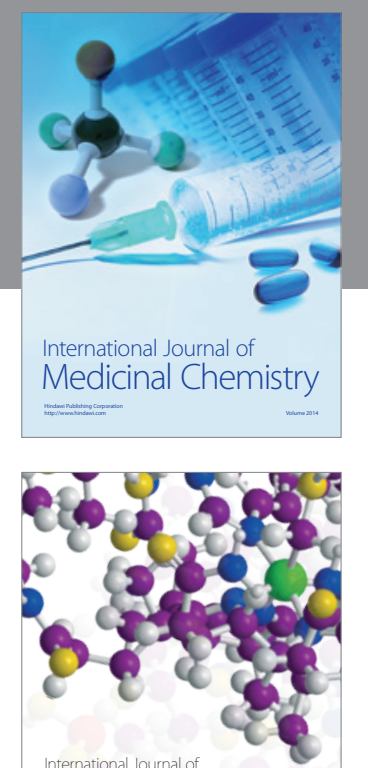

\section{Carbohydrate} Chemistry

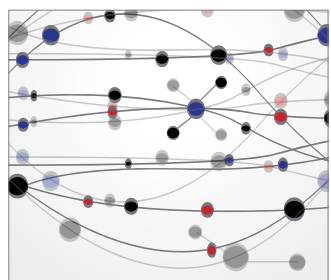

The Scientific World Journal
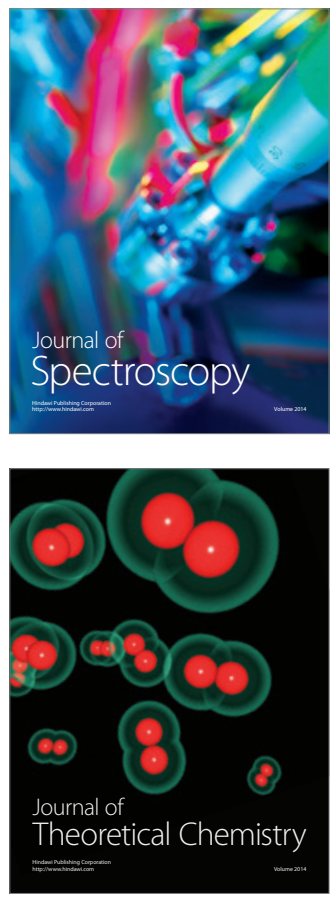
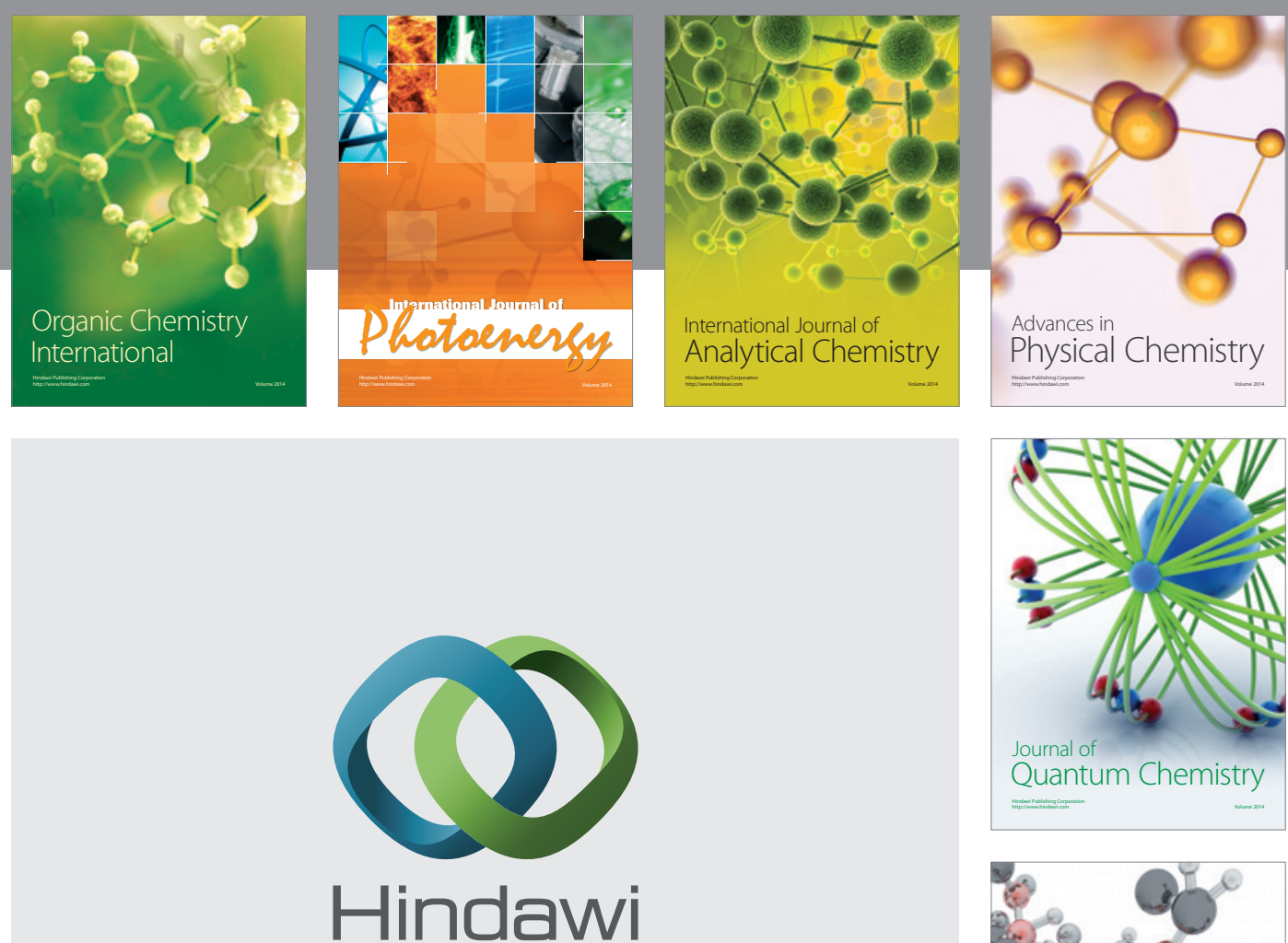

Submit your manuscripts at

http://www.hindawi.com

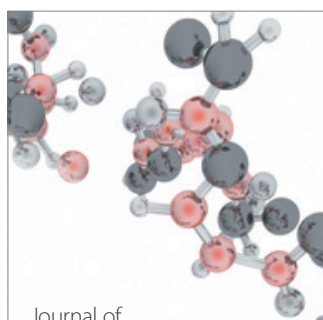

Analytical Methods

in Chemistry

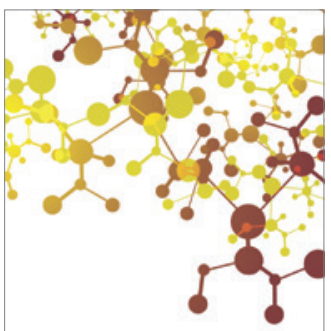

Journal of

Applied Chemistry

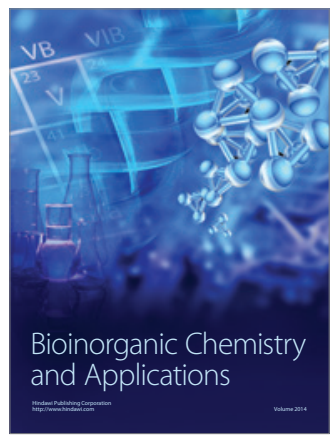

Inorganic Chemistry
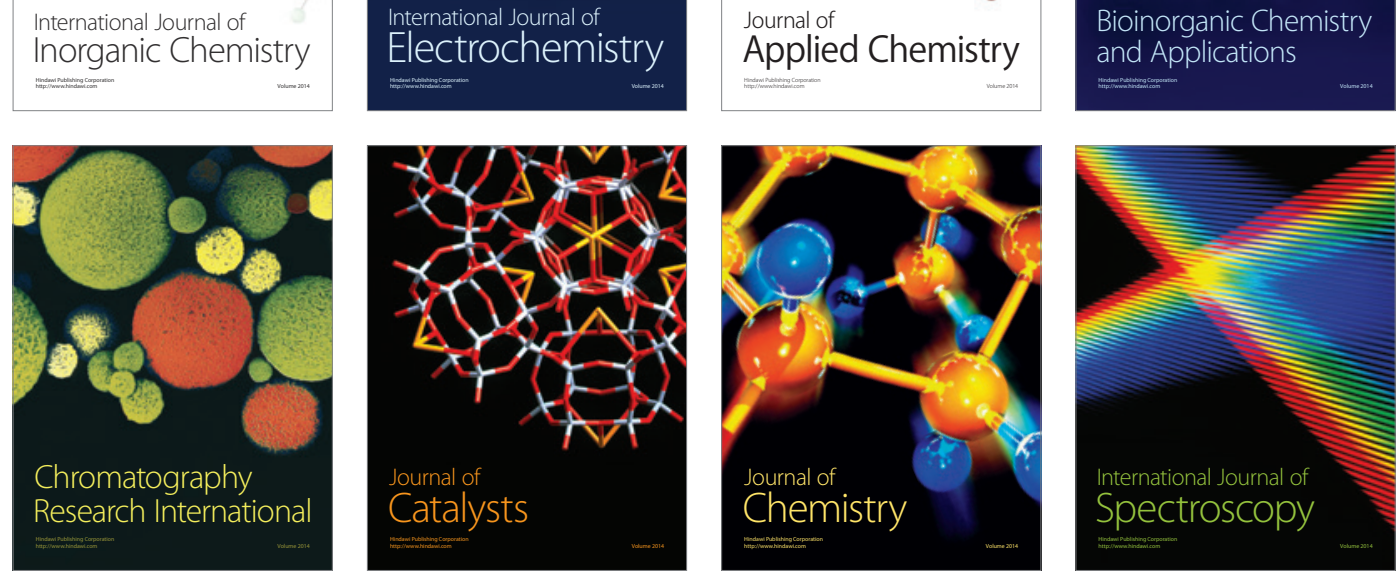